\title{
Second-order Nonlinear Analysis of Aluminium Alloy Frame
}

\author{
Xiawei Tan1, a , Zheng Zhang1, b, Juanjuan Huang ${ }^{1, c}$ and Rensheng Liao 1,d \\ ${ }^{1}$ School of Civil Engineering, Fujian University of Technology, No.33 Xue Fu South Road, \\ University Town, Fuzhou, Fujian, P.R. China, 350118 \\ atanxiawei_fz@163.com,bfrp_creep@sina.com, chjj850382@163.com, ${ }^{d} 18060592156 @ 163 . c o m$
}

Keywords: aluminium alloy frame, nonlinear analysis, finite element analysis

Abstract. Aluminum alloy is widely used in civil engineering because of the advantages of light weight and high strength. But the elastic modulus of aluminum alloy is only one-third of steel, and the deformation of aluminum alloy is more notable. Second-order nonlinear finite element analysis is performed to analyze a multilayer aluminum alloy frame. Precise second-order nonlinear analysis is compared with the approximate second-order analysis method which is adopted by the Chinese structural aluminum alloy design code GB50429. The approximate second-order analysis method adopted by the code are found partial to be unsafe when material nonlinearity being considered. It is recommanded that the current frame design method be modified to provide a safe and reliable design for Aluminum alloy frame considering both geometric and material nonlinearities .

\section{Introduction}

Aluminium alloy materials have many advantages such as light, high strength, good corrosion resistance and good plasticity, which are the reasons why aluminium alloy structures are widely used in civil engineering. At present, several countries have incorporated the second-order theory into structural aluminum alloy design codes [1-3]. The formulas for second-order effect of the frame structure of the GB50429 [3] are based on the second-order elastic analysis, which are the same as the Chinese structural steel design code GB50017 [4]. However, the aluminum alloy has a significant residual deformation before its carrying capacity reaches the nominal yield strength. At the same time, the steel with the same yield strength is still in its elastic deformation stage. In this range, if it is still designed according to the elastic method whether there will be greater error and insecurity, which needs further study. In view of this situation, the large-scale general finite element software ABAQUS is used to analyze second-order nonlinear behaviors of a typical multilayer aluminum alloy frame structure. Through comparison with the approximate second-order analysis method, to discuss the applicability of GB50429 on the second-order analysis directly follow GB50017 relevant formulas, and then some suggestions on aluminum alloy frame structure analysis and design are put forward.

\section{Analysis Methods of Aluminum Alloy Frame Structure}

The first-order analysis is a balanced analysis of the undeformed structure. When the structure has no side shift or the second-order calculation method is designed partial to be safe, the first-order calculation method can be used to analyze the whole structure. Except these cases, the analysis of frames needs to conduct second-order analysis [2]. When using second-order linear analysis, Consideration should be given to the effects of various defects in the structure and members (such as the initial tilt and initial eccentricity of the column) on internal forces, I.e. geometric nonlinearity. For the stability of frame structure, in principle it is ought to use second-order nonlinear analysis. That is, in addition to considering the geometric nonlinearity, the material nonlinearity also should to be considered. Therefore, the second-order effect of aluminum alloy structure according to its true stress-strain relationship should be carried out. 


\section{Finite Element Analysis Method}

The frame is calculated by using the linear beam element B21 in ABAQUS. Based on the Timoshenko beam theory, the influence of transverse shear deformation is considered in addition to the deformation such as axial, bending and torsion. It is suitable for structural analysis of large strain and large deformation.

For the geometric nonlinearity, the large deformation switch is required to be opened in the analysis step. At the same time, the initial roll of the aluminum alloy frame is $H / 1000(\mathrm{H}$ is the total height of the frame) as the initial geometrical defect of the whole structure [5]. The analysis did not take into account the semi-rigid, the column feet and nodes are set to rigid connection.

The stress-strain curve of aluminium alloy adopt Ramberg-Osgood model [6], which are verified to be appropriate for aluminium alloys structure analysis.

\section{Second-order Nonlinear Analysis}

The internal forces of the aluminum alloy frame are calculated and analyzed by the second-order nonlinear analysis and the approximate formulas recommended by GB50429. The concrete example and analysis results are as follows.

Figure 1 is the 2-bay 5-story aluminum alloy frame calculation sketch, the frame section size and its section characteristics are shown in table 1. The elastic modulus of aluminium alloy was set to 70 $\mathrm{GPa}$, and Poisson's ratio was set to 0.3 . The nominal yield strength of aluminium alloy was set to 240 MPa. By adjusting the ratio of the horizontal load $H$ to the vertical load $q$, the $\Sigma N \cdot \Delta u /(\Sigma H \cdot h)$ of the aluminum alloy frame is $0.06,0.08 \ldots 0.28$, respectively. When $\Sigma N \cdot \Delta u /(\Sigma H \cdot h)$ are $0.06,0.08,0.10 \ldots$ $0.24,0.25,0.26$ and $0.26, q$ are $40,53.3,66.7,80,93.3,106.7,120,133.3,146.7,160,167,173.3$, $186.7 \mathrm{kN} / \mathrm{m}$, respectively.

Table 1. Section properties of beams and columns of 2-bay 5-story frame

\begin{tabular}{cccc}
\hline Section & Section types & $\mathrm{A} / \mathrm{m}^{2}$ & Moment of inertia $/ \mathrm{m}^{4}$ \\
\hline Left beam & HN500 $\times 200 \times 10 \times 16$ & $1.14 \times 10^{-2}$ & $4.78 \times 10^{-4}$ \\
Right beam & HN600 $\times 200 \times 11 \times 17$ & $1.35 \times 10^{-2}$ & $7.82 \times 10^{-4}$ \\
Side column & HW350 $\times 350 \times 12 \times 19$ & $1.74 \times 10^{-2}$ & $4.03 \times 10^{-4}$ \\
Middle column & HW400 $\times 400 \times 13 \times 21$ & $2.20 \times 10^{-2}$ & $6.69 \times 10^{-4}$ \\
\hline
\end{tabular}

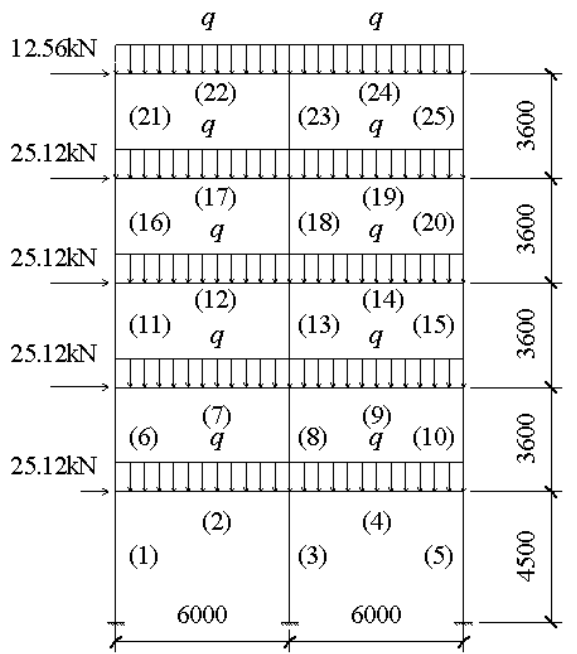

Fig 1. Calculation sketch of 2-bay 5-story aluminum alloy frame

It can be seen from Fig. 2 that when $\Sigma N \cdot \Delta u /(\Sigma H \cdot h)$ ratio is 0.1 and the yield stress $\sigma_{\max }=1 / 2 f_{0.2}$, the difference between the second-order elasticity analysis and the second-order nonlinear analysis is so obvious that should not be neglected. With the increase of $\sigma_{\max }$, the difference between the second-order elasticity analysis and the second-order nonlinear analysis is getting bigger and bigger. 
From $\sigma_{\max }=1 / 2 f_{0.2}$ to $\sigma_{\max }=f_{0.2}$, the difference between the 2-bay 5- story framework is from $20 \%$ to $146 \%$. Therefore, it is recommended GB50429 on the $\sigma_{\max }$ taken as $1 / 2 f_{0.2}$.

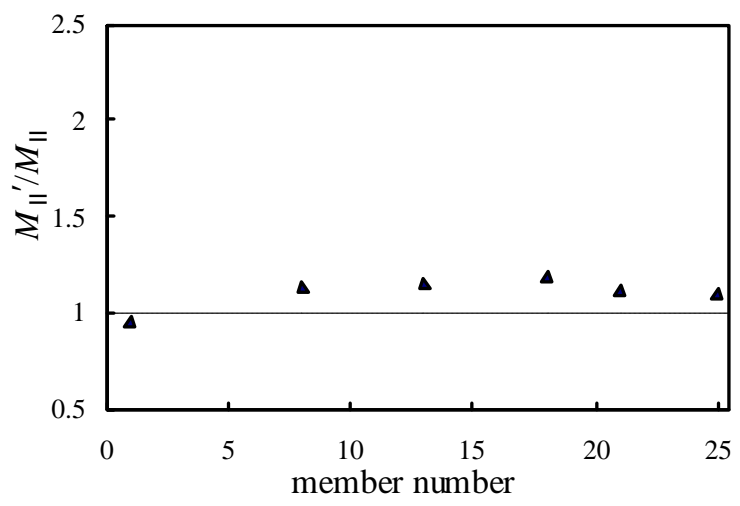

(a) Column end moment $\left(\sigma_{\max }=1 / 2 f_{0.2}\right)$

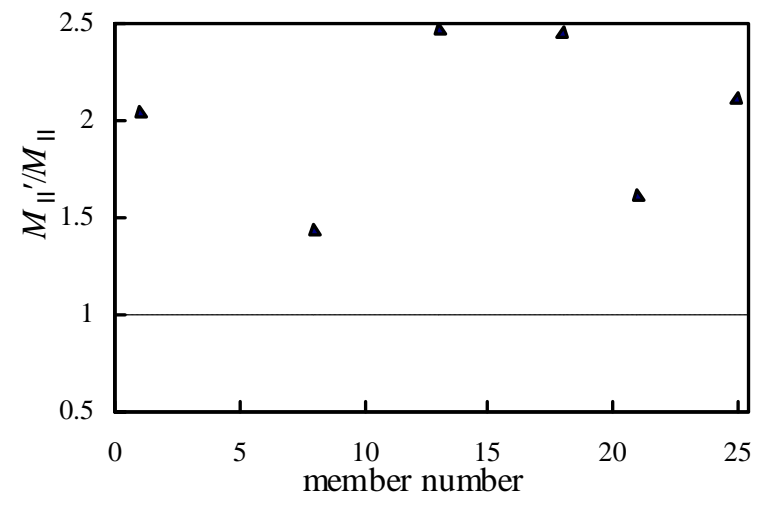

(b) Column end moment $\left(\sigma_{\max }=f_{0.2}\right)$

Notes: The ordinate in the graph is the ratio of the bending moment $\left(M_{\text {II }}\right)$ of the second order elastic-plastic analysis to the bending moment $\left(M_{\text {II }}\right)$ calculated by the approximate second-order analysis, the same below.

Fig 2. Column end moment ratio of 2-bay 5-story aluminum alloy frame

When the internal force analysis is taken as $\sigma_{\max }=1 / 2 f_{0.2}$, it can be seen from Fig. 3 that in the first stage, when $\Sigma N \cdot \Delta u /(\Sigma H \cdot h)$ is between 0.06 and 0.1 , the differences between second-order elastic-plastic analysis and second-order elastic analysis is from $1.40 \%$ to $4.90 \%$, which are so small that it could be neglected. Therefore, when $\Sigma N \cdot \Delta u /(\Sigma H \cdot h) \leq 0.1$, the second-order elastic analysis can be used to calculate the internal force of frames, which is consistent with the rules of GB50429. In the second stage, with the increase of $\Sigma N \cdot \Delta u /(\Sigma H \cdot h)$, the ratio of the bending moment of the second-order elastic-plastic analysis to the bending moment of the second-order elastic analysis is also increased. When the $\Sigma N \cdot \Delta u /(\Sigma H \cdot h)$ is 0.25 , the gap between the two up to $91 \%$, which is partial to unsafe, and should not be ignored, therefore it is recommended the GB50429 to be amended to meet the safety and design meets. In the third stage, when the $\Sigma N \cdot \Delta u /(\Sigma H \cdot h)$ increases from 0.25 to 0.26 and 0.28 respectively, the ratio of the bending moment of the two analysis increases sharply, and the maximum increases are $27.8 \%$ and $46.4 \%$ respectively, which in the structural design is not allowed to appear. At this time GB50429 will no longer apply, but GB50429 also made the relevant provisions, that is, when the $\Sigma N \cdot \Delta u /(\Sigma H \cdot h)>0.25$, the use of approximate design method error is large, this time should be appropriate to increase the lateral stiffness of the structure. At the same time, it can be seen from Fig. 3 that it is reasonable to carry out the second-order analysis of frame structure in GB50429 with $0.1<\Sigma N \cdot \Delta u /(\Sigma H \cdot h) \leq 0.25$, i.e. the second stage. Since the stresses of beams are quite small and no control effect and the dispersion are large, this paper only selects the bending moment of columns for comparative analysis.

\section{Conclusions}

Through the calculation and analysis of the paper, the following conclusions can be drawn:

(1) Because of the notable nonlinearity of the aluminum alloy, when the yield stress is $\sigma_{\max }=1 / 2 f_{0.2}$, the difference between second-order elastic analysis and second-order nonlinear analysis is very obvious, which should not be overlooked.

(2) it is reasonable to carry out the second-order analysis of frame structure in GB50429 with 0.1 $<\Sigma N \cdot \Delta u /(\Sigma H \cdot h) \leq 0.25$, i.e. the second stage.

(3) In consideration of the material nonlinearity of Aluminum Alloy will have a great impact on the frame of internal force calculation. When $0.1<\Sigma N \cdot \Delta u /(\Sigma H \cdot h) \leq 0.25$, with the increase of the ratio of $\Sigma N \cdot \Delta u /(\Sigma H \cdot h)$, the differences between second-order nonlinear and second-order elastic internal 
force analysis increases from $4.9 \%$ to $91 \%$, which are partial to unsafe, and should not be ignored, and if the influence is neglected, the internal force calculation result will be too small and the design

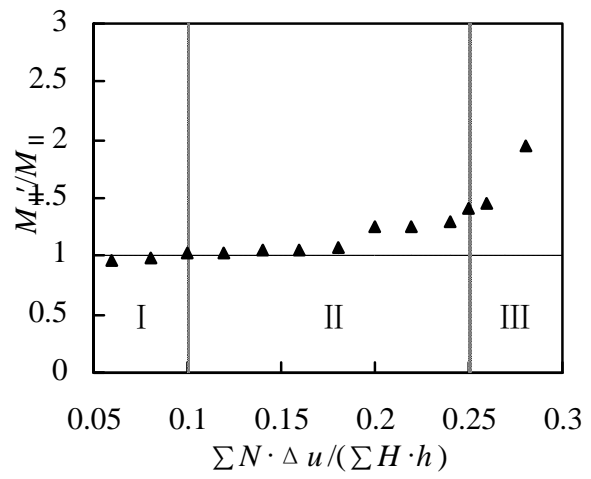

(a)member 1

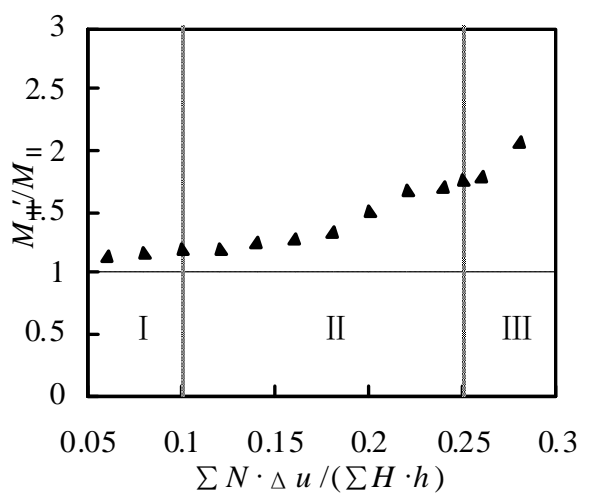

(d)member 18

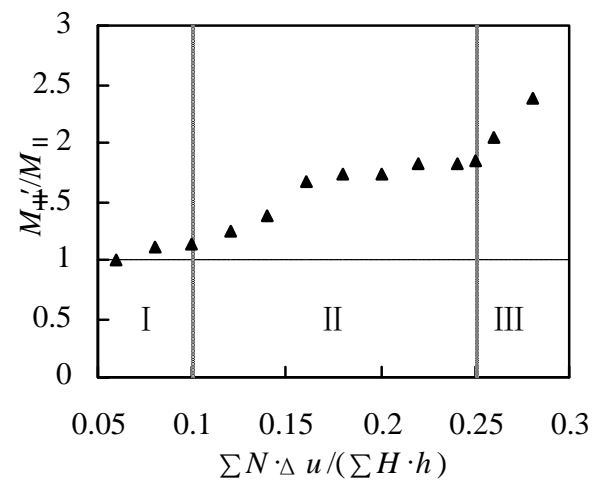

(b) member 8

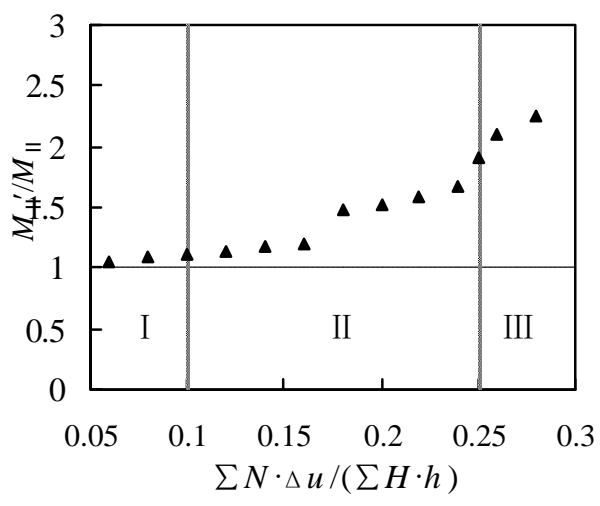

(e) member 21

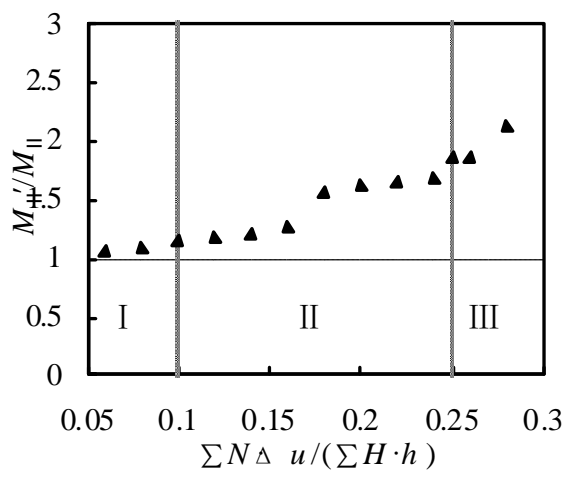

(c) member 13

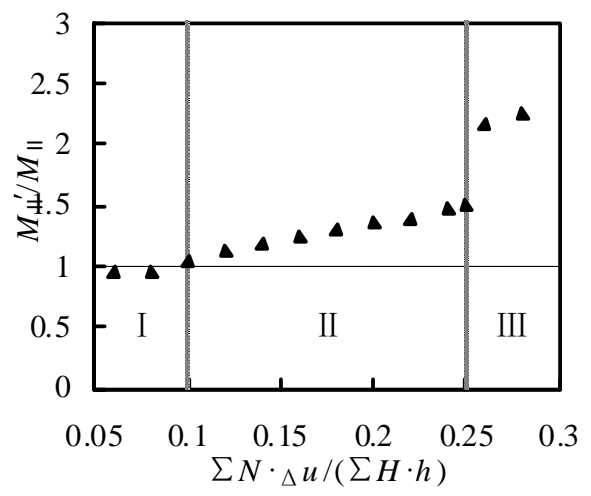

(f) member 25

Fig 3. Internal force analysis results of 2-bay 5-story aluminum alloy frame

will be unsafe. Therefore, It is necessary to continue the study and put forward relavant design proposals or modify relavant formulas at that range.

\section{Acknowledgments}

This work was financially supported by National Natural Science Foundation of China (51208109), National Natural Science Foundation of Fujian Province (2017J01670) and Key Project of Fujian College Youth Natural Fund (JZ160465).

\section{References}

[1] Aluminum Association. Aluminum design manual: specifications \& guidelines for aluminum structures. Arlington: Aluminum Association Inc, 2005.

[2] Eurocode 9: Design of aluminium structures-Part 1-1: General structural rules. European Committee for Standarization. 2007. p. 11..

[3] National standards of P.R.C: Code for the design of aluminium structures GB 50429-2007.

[4] National standards of P.R.C: Code for the design of steel structures GB 50017-2003.

[5] Liu J. Second-order Inelastic Theory and Application of Higher Analysis of Steel Structures (Science Press, Beijing 2012).

[6] M.M. Federico: Aluminium alloy structures (Chapman \& Hall, London 1995). 